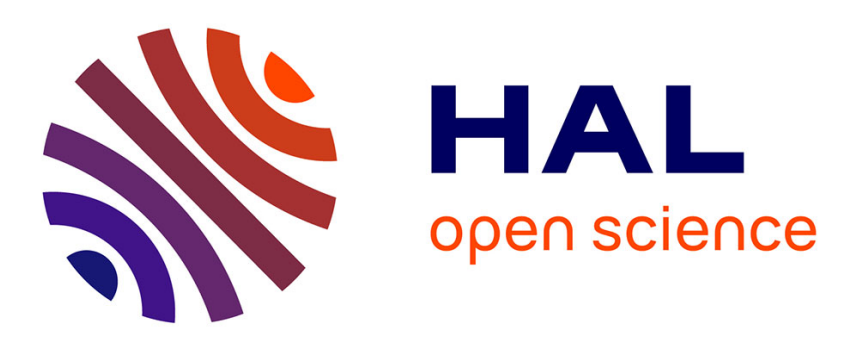

\title{
CARACTÉRISATION DE SOLUTIONS AQUEUSES DE GLUCIDES PAR ULTRASONS HAUTES FRÉQUENCES
}

\author{
M. Asmani, Edouard Radziszewski, Malika Toubal, Mohamed Ourak, \\ Bertrand Nongaillard
}

\section{To cite this version:}

M. Asmani, Edouard Radziszewski, Malika Toubal, Mohamed Ourak, Bertrand Nongaillard. CARACTÉRISATION DE SOLUTIONS AQUEUSES DE GLUCIDES PAR ULTRASONS HAUTES FRÉQUENCES. Journal de Physique IV Proceedings, 1992, 02 (C1), pp.C1-911-C1-914. 10.1051/jp4:19921199 . jpa-00251164

\section{HAL Id: jpa-00251164 https://hal.science/jpa-00251164}

Submitted on 1 Jan 1992

HAL is a multi-disciplinary open access archive for the deposit and dissemination of scientific research documents, whether they are published or not. The documents may come from teaching and research institutions in France or abroad, or from public or private research centers.
L'archive ouverte pluridisciplinaire HAL, est destinée au dépôt et à la diffusion de documents scientifiques de niveau recherche, publiés ou non, émanant des établissements d'enseignement et de recherche français ou étrangers, des laboratoires publics ou privés. 


\title{
CARACTÉRISATION DE SOLUTIONS AQUEUSES DE GLUCIDES PAR ULTRASONS HAUTES FRÉQUENCES
}

\author{
M. ASMANI, E. RADZISZEWSKI, M. TOUBAL, M. OURAK et B. NONGAILLARD \\ Laboratoire d'Opto-Acousto-Electronique, URA 832 CNRS, Université de Valenciennes, BP. 311, \\ F-59304 Valenciennes, France
}

\begin{abstract}
Résumé: Le but de notre travail est de déterminer linfluence de la structure et de la concentration de glucides simples (oses) et composés (diholosides) sur l'atténuation ultrasonore de solutions contenant ce type de molécules. Les expériences montrent qu'il n'existe aucune différence d'atténuation entre une solution aqueuse de glucose (aldose) et une solution de fructose (cétohexose) dans les mêmes concentrations molaires. Par contre les solutions aqueuses de saccharose (glucose-fructose) et de maltose (glucose-glucose) présentent des valeurs d'atténuation différentes surtout aux basses fréquences (40-150 MHz). D'autre part, l'influence de la concentration des glucides sur l'absorption ultrasonore haute fréquence (50-500 MHz) a été mise en évidence au cours de nos travaux.
\end{abstract}

\begin{abstract}
Our aim is to investigate the influence of the structure and concentration of simple and compound sugars on the ultrasonic absorption of solutions containing these molecules. Our experiments show no difference between the attenuation of an aqueous solution of glucose and a fructose one with the same molar concentrations. However aqueous solutions of sucrose (glucose-fructose) and maltose (glucose-glucose) exhibit different attenuation figures, mainly at low frequencies (40-50 MHz). Morever the influence of the sugars concentration on ultrasonic absorption has been evidenced at frequencies between 50 and $500 \mathrm{MHz}$.
\end{abstract}

\section{I-INTRODUCTION}

Le rôle des biomolécules dans le mécanisme d'atténuation des ultrasons fait actuellement l'objet de très nombreux travaux. Parmi ces molécules, les protéines, constituants principaux des tissus biologiques, sont les plus étudiées [1], [1]'. Pour notre part, nous nous sommes attachés à montrer l'influence, dans les phénomènes d'absorption ultrasonore haute fréquence $(50-500 \mathrm{MHz})$, de la structure d'autres macrobiomolécules participant également à l'élaboration de la matière vivante : les glucides, simples et composés.

\section{II-ABSORPTION DES ULTRASONS DANS LES SOLUTIONS AQUEUSES}

\section{A-INTRODUCTION}

BIQUARD [2], fut le premier à constater que les ultrasons sont absorbés par les liquides. KNESER [3], justifia ceci par la relaxation thermique. TOX et ROCK [4] mesurèrent l'absorption de l'eau entre 2 et $40^{\circ} \mathrm{C}$ et constatèrent un comportement particulier à $4^{\circ} \mathrm{C}$, ils rejetèrent alors l'hypothèse de Kneser et proposèrent la relaxation de la structure.

\section{B-CLASSIFICATION DES LIQUIDES}

En 1948, Pinkerton [5] fit une classification des liquides en fonction de leurs propriétés ultrasonores d'absorption. Il-distingua alors deux types de liquides :

- Les liquides normaux (liquides monoatomiques : $\mathrm{O}_{2}, \mathrm{H}_{2}, \mathrm{~N}_{2} \ldots$ ).

- Les liquides anormaux, qui se subdivisent en : 
- Liquides de Kneser dont l'absorption est due à la lenteur d'échange d'énergie interne et externe de la molécule. Ce groupe comprend quelques liquides inorganiques et la majorité des groupes organiques.

- Liquides "associés", dont le comportement est semblable à celui de l'eau.

\section{C-PHENOMENES RESPQNSABLES DE L'ATTENUATION [6]}

La propagation d'une onde ultrasonore dans un milieu quelconque (solide ou liquide), s'accompagne toujours d'une atténuation, liée à la fréquence de l'onde. Suivant les dimensions des particules constituant le milieu, par rapport à la longueur d'onde acoustique, on peut mettre en évidence deux effets :

- Atténuation due à l'absorption

- Atténuation due à la diffusion

Pour les liquides associés, la contribution de la diffusion est négligeable, par conséquent l'atténuation est due essentiellement à l'absorption. Ce qui permet de faire une analyse suivant deux modèles :

1) Modèle de la molécule rigide ou indéformable (liquides associés).

a) En basse fréquence

Du point de vue phénoménologique, il peut être mis en évidence deux types d'absorption :

- Absorption par thermoconduction (uniquement pour les ondes longitudinales).

- Absorption par frottements internes : viscosité.D'une façon générale, la fonction de dissipation $\Psi$ (énergie dissipée par unité de temps et de volume), se met sous la forme :

$$
\psi=\frac{1}{2} \eta_{\mathrm{ijk} k} \varepsilon_{\mathrm{ij}} \varepsilon_{\mathrm{kl}}+\kappa_{\mathrm{ik}} \frac{\partial^{2} \mathrm{~T}}{\partial \mathrm{x}_{\mathrm{i}} \partial \mathrm{x}_{\mathrm{k}}}
$$

où : $\eta_{\mathrm{ijkl}}$ est le tenseur de viscosité.

$\mathrm{T}$ : température.

$\kappa_{\mathrm{ik}}$ est le tenseur de conductivité thermique.

$\varepsilon_{\mathrm{ij}}$ est le tenseur de déformation.

b) En haute fréquence: phénomènes de relaxation

La notion de relaxation est reprise par plusieurs auteurs, pour expliquer la forte absorption que présentent certains milieux. D'une façon générale, un processus de relaxation pour un système est un phénomène, lié au retour à l'état d'équilibre. Zener [7], a montré que pour un milieu perturbé par une onde ultrasonore haute fréquence, la loi de Hooke n'est plus valable, elle est remplacée par une relation générale de la forme :

$$
\sigma_{\mathrm{ik}}+\tau \dot{\sigma}_{\mathrm{ik}}=\mathrm{C}_{\mathrm{iklm}} \varepsilon_{\mathrm{lm}}+\tau \mathrm{C}_{\mathrm{iklm}}^{\prime} \dot{\varepsilon}_{\mathrm{lm}}
$$

$\tau:$ temps de relaxation.

$\mathrm{C}_{\mathrm{iklm}}$ et $\mathrm{C}_{\mathrm{iklm}}^{\prime}$ : constantes élastiques non relaxées et relaxées.

$\sigma_{\mathrm{ik}}:$ tenseur de déformation .

Ce qui permet de distinguer :

- La relaxation thermique.

- La relaxation des constantes élastiques ou de viscosité.

Devault [8], a montré que la viscosité est liée aux constantes de Grüneisen, elles mêmes liées aux constantes élastiques. 


\section{2) Modèle de la molécule déformable ou "pulsée" (liquide de Kneser).}

Dans le cas de la molécule "pulsée", aux effets thermiques et à la viscosité viendra s'ajouter une absorption moléculaire, due aux échanges d'énergie entre la molécule et ses atomes, qui sera aussi caractérisée par une relaxation moléculaire.

D'une façon générale la contribution des effets thermiques est négligeable devant les effets de viscosité.

\section{EXPERIMENTATION ET RESULTATS}

Les mesures sont faites par transmission d'ondes longitudinales, dans la gamme de fréquence (50-500 $\mathrm{MHz}$ ) à température ambiante $\mathrm{T}=22^{\circ} \mathrm{C}$. Le déplacement micrométrique de l'un des deux transducteurs permet de mesurer l'atténuation. Les échantillons utilisés sont des glucides en solution :

- oses (glucose, fructose).

- osides (saccharose, maltose).

La figure 1, montre que l'atténuation d'une solution aqueuse, d'un mélange de glucose et fructose à la concentration de $2.78 \mathrm{~mol} / \mathrm{l}$ est sensiblement identique à celle d'une solution contenant uniquement du glucose ou du fructose à la même concentration, quelque soit la fréquence utilisée. La différence de structure moléculaire entre les deux oses considérés, glucose (aldohéxose) et fructose (cétohéxose), ne semble pas avoir d'influence sur l'atténuation ultrasonore haute fréquence.

La solution de saccharose (glucose-fructose) à la concentration de $2.78 \mathrm{~mol} / \mathrm{l}$ présente une atténuation beaucoup plus importante que la solution contenant le mélange (glucose+fructose) à la même concentration (figure 3.a). Il en est de même pour la solution de maltose (glucose-glucose), comparée à celle du glucose dans les mêmes concentrations molaires (figure 3.b). Les mesures d'absorption de solutions d'oses et d'osides montrent que les osides présentent une atténuation plus importante que les solutions d'oses dont ils sont constitués [1]. Par conséquent, la formation de nouvelles liaisons $\mathrm{O}$ glycosidiques entre les monomères influe considérablement sur l'atténuation ultrasonore. La comparaison entre les courbes d'atténuation des solutions de saccharose (glucose-fructose) et de maltose (glucose-glucose) dans les mêmes concentrations (figure 4), montre l'importance de la structure moléculaire des diholosides dans le phénomène d'atténuation ultrasonore surtout dans la gamme de fréquences $(40-150 \mathrm{MHz})$.

\section{CONCLUSION}

L'étude d'oses (glucose, fructose) et d'osides (saccharose, maltose) en solution, nous a permis de constater l'influence de leur masse, de leur concentration et de leur structure moléculaire sur l'atténuation ultrasonore haute fréquence. Actuellement notre travail se poursuit sur d'autres types de molécules glucidiques : trioses, pentoses, osamines, acides uroniques. Parallèlement, une modélisation au niveau moléculaire de l'absorption des ultrasons en hautes fréquences dans les solutions aqueuses et dans les milieux biologiques, par intéractions phonons-phonons est en cours.

\section{REFERENCES}

[1] F.W. Kremkau "Biomolecular absorption of ultrasound.I : Molecular weight". J.A.S.A 76, 1330-1335 (1984).

[1'] F.W Kremkau and R.W. Comgill. " Biomolecular absorption of ultrasound.II : Molecular Structure".

J.A.S.A 77, 1217-1221 (1984).

[2] P. Biquart, Compt. rend 193,226 (1931); 309 (1933).

[3] H. O. Kneser, Ann. Physik [5]. 32, 277 (1938).

[4] F. E. Fox and G. D. Rock, Phys. Rev. 70, 68 (1946).

[5] J. Lamb and J. M. M. Pinkerton, Proc. Roy. Soc A 199, 114 (1949).

[6] A. Dahel Thèse de Doctorat Es-Sciences Soutenue 1984 à l'Université d'Alger.

[7] Zener. "Elasticity and Aelasticity of metals", University of Chicago, Chicago-Press (1948).

[8] G.P. Devault Physical Review. Vol 155 Nb 3 (March 1967). 


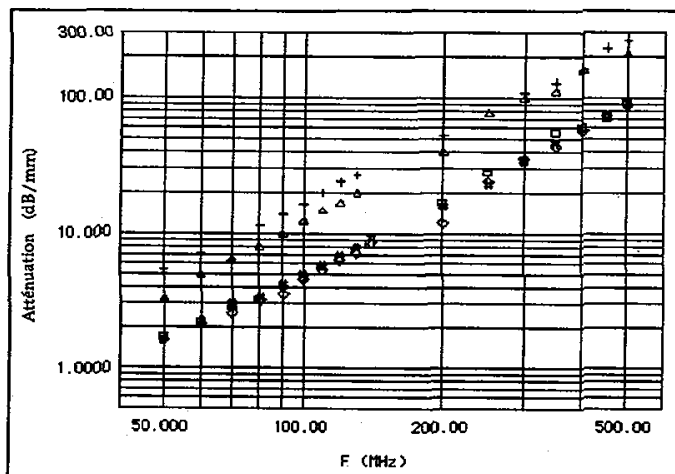

Figure 1: Loi de variation de l'atténuation en fréquence des oses en solution et des diholosides en solution à la concentration de $2.78 \mathrm{~mol} / \mathrm{h}$.

+ : Matose; $\diamond:$ Fructose; $\square$ : Glucose; $\Delta:$ Saccharose;

: Glucose + Fructose.

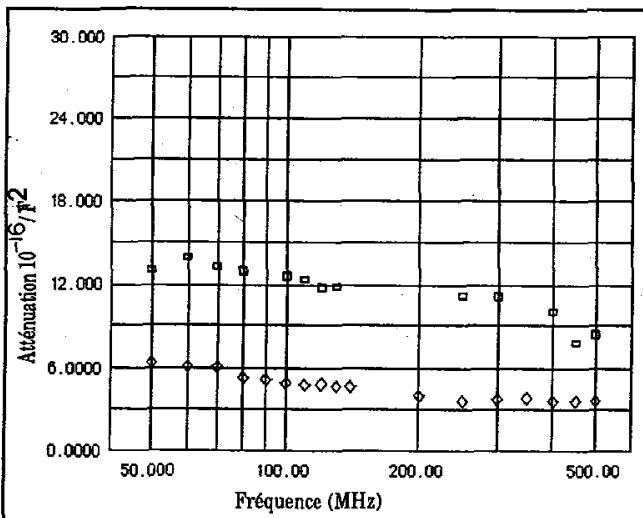

(a) $\square$ Saccharose $2,78 \mathrm{~mol} / \quad \diamond$ mélange glucose+fructose $2,78 \mathrm{~mol} /$

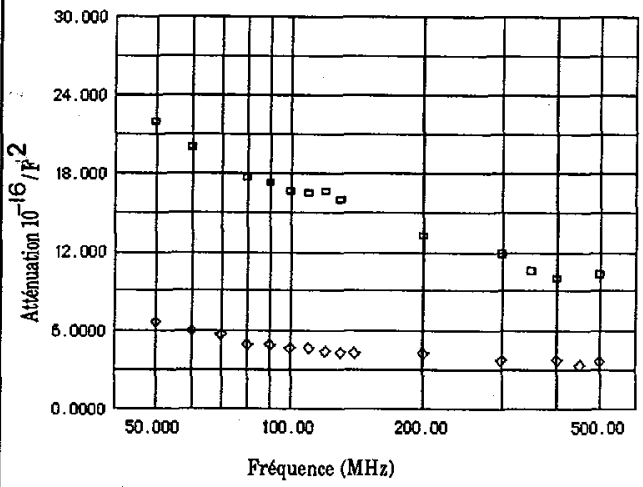

(b) $\square$ maltose $2,78 \mathrm{~mol} / \mathrm{h} \diamond$ glucose $2,78 \mathrm{~mol} / \mathrm{h}$

Figure 3: Comparaison entre l'atténuation de solutions de diholosides ( saccharose, maitose) et de solutions contenant les oses dont ils sont formés ( glucose, fluctose ) à concentration égale
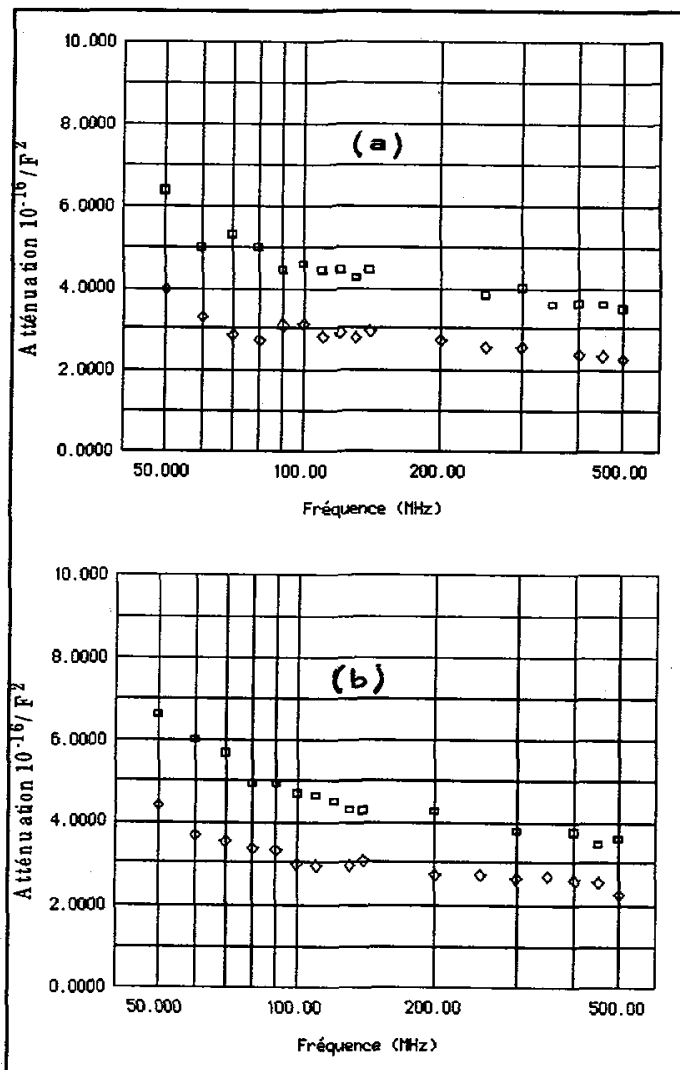

Figure 2: Influence de la concentration des oses (fructose, glucose) sur l'atténuation en fonction de la fréquence.

(a) fructose, (b) glucose.

ㅁ : $2.78 \mathrm{~mol} / \mathrm{l} ; \bullet: 1.11 \mathrm{~mol} / \mathrm{l}$.

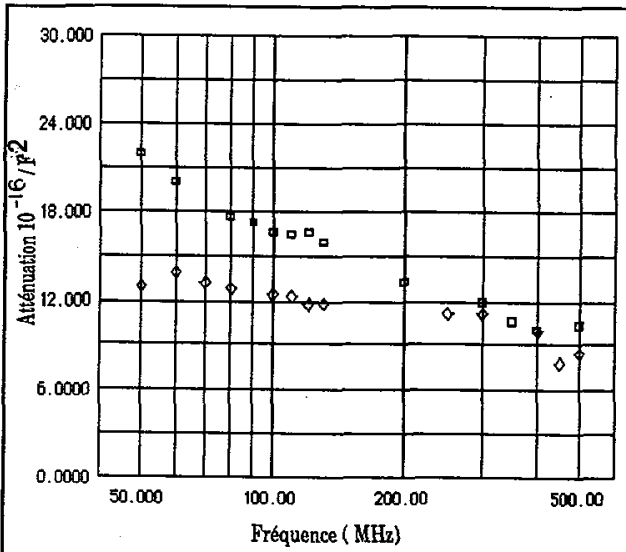

a saccharose 2,78 moll $\diamond$ maltose 2,78 moln

Figure 4: Comparaison entre l'atténuation de deux solutions de diholosides ( maltose, saccharose) en fonction de la fréquence. 